\title{
Cardiac Shock Wave Therapy Attenuates Cardiomyocyte Apoptosis after Acute Myocardial Infarction in Rats
}

\author{
Yunhe Zhang ${ }^{a, b, d}$ Tao Shen ${ }^{c}$ Bing Liu ${ }^{b}$ Dapeng Daic Jianping Caic \\ Cong Zhao ${ }^{b}$ Ling Du ${ }^{b} \mathrm{Na} \mathrm{Jia}^{\mathrm{b}}$ Qing He $\mathrm{He}^{\mathrm{a}, \mathrm{b}}$ \\ ${ }^{a}$ Graduate School of Peking Union Medical College and Chinese Academy of Medical Sciences, Beijing, \\ ${ }^{b}$ Department of Cardiology, Beijing Hospital, National Center of Gerontology, Beijing, 'The $\mathrm{MOH}$ \\ Key Laboratory of Geriatrics, Beijing Hospital, National Center of Gerontology, Beijing, 'Department \\ of Geriatric \& VIP, Sichuan Academy of Medical Sciences and Sichuan Provincial People's Hospital, \\ Chengdu, Sichuan, China
}

\section{Key Words}

Shock wave therapy $\cdot$ Acute myocardial infarction $\cdot$ Apoptosis $•$ JNK

\begin{abstract}
Background/Aims: Researches have showed that cardiac shock wave therapy (CSWT) could improve left ventricular function and attenuate LV remodeling of the ischemic heart. Apoptosis plays an important role in myocardial infarction and determines heart function and prognosis. However, it is still not clear whether CSWT is sufficient to attenuate acute myocardial infarction (AMI) induced cardiomyocyte apoptosis in vivo. In this study, we used a rat model to examine whether CSWT could attenuate cardiomyocyte apoptosis after AMI and to explore potential mechanisms. Methods: We generated an AMI rat model to investigate the function and possible regulatory mechanisms of CSWT. All rats were randomly divided into four groups: the sham-operated only group, sham-operated with SW treatment group, AMI only group, and AMI treated with SW treatment group.The rats were treated with a left anterior descending coronary artery ligation for $12 \mathrm{~h}$ and then treated with or without CSWT (800 shots at $0.1 \mathrm{~mJ}$ / $\mathrm{mm}^{2}$ ). Cytochrome $\mathrm{c}$ release was measured to analyze mitochondrial function and integrity. The apoptotic cell rate was determined by TUNEL assay. Western blot was used to analyze the cell apoptosis-, inflammation-, and survival-related signaling pathways. Results: First, the methodology of CSWT in the rat model of AMI was established. Second, CSWT attenuated the cardiomyocyte apoptosis rate in the infarct border zone. Third, CSWT suppressed the expression of apoptosis and inflammation molecules after AMI. Fourth, CSWT inhibited activation of the JNK pathway, which indicated inhibition of the cell inflammatory pathways and promotion of cardiomyocyte survival after AMI. Conclusion: These results indicate that CSWT exerts a protective effect against AMI-induced cardiomyocyte apoptosis, potentially by
\end{abstract}


attenuating cytochrome $\mathrm{c}$ release from the mitochondria and inhibiting of the mitochondrialdependent intrinsic apoptotic pathway. We also demonstrate that CSWT suppresses the JNK pathway and cardiomyocyte inflammation, which may also decrease cardiomyocyte apoptosis in vivo.

\section{Introduction}

Coronary heart disease (CHD) is the major cause of morbidity and mortality in developed countries. Medical treatment, percutaneous coronary intervention (PCI), and coronary artery bypass grafting (CABG) are leading available therapeutic options for chronic CHD. The prognosis of patients suffering from advanced CHD without indications for PCI or CABG is still poor despite optimal medical therapy. Therefore, it is considerable to explore alternative therapeutic methods for these patients.

Acute myocardial infarction (AMI) accompanies with a loss of cardiomyocytes and is followed by development of heart failure and poor prognosis [1]. It is known that apoptosis is the vital pathogenesis of myocardial infarction (MI). Studies have demonstrated that cardiomyocyte apoptosis takes place in heart samples from patients suffering from MI [2]. Inhibition of cardiomyocyte apoptosis MI-induced can lead to a decrease of the infarct size and an improvement of cardiac function [3-5]. Cardiac shock wave therapy (CSWT) is a novel approach that provides a new choice for severe CHD. In recent several years, many of in vitro and vivo studies have shown that low energy shock wave (SW) induces a "cavitation effect" that is defined by a micrometer-sized vehement burst of bubbles within and outside of cells [6,7]; the burst exerts a mechanical shear force on heart tissue and endothelial cell membranes to exert biological effects with following up-regulation of VEGF and eNOSin ischemic myocardium $[8,9]$ to promote angiogenesis and improve myocardial perfusion $[10$, $11]$.

Recent studies have demonstrated that CSWT can improve left ventricular (LV) function and attenuate LV remodeling of the ischemic heart via the following mechanisms: enhancing angiogenesis, promoting EPC homing, reducing oxidative stress, alleviating inflammation, and preserving mitochondrial function. Apoptosis is one of the most key pathogenic mechanisms of CAD due to loss of cardialmyocyte. Studies have demonstrated that patients with MI and advanced congestive heart failure (CHF) associate with higher rates of cardiomyocyte apoptosis than normal ones. Nonetheless, it is not clear whether CSWT has any benefit on cardiomyocyte apoptosis. Based on promising results from clinical and animal trials, our team adopted a H9c2 cellline ischemia/hypoxia $(\mathrm{I} / \mathrm{H})$ model to observe the impacts of SW therapy on apoptosis in vitro. The results showed the potential benefits of shock wave treatment against I/H-induced cell apoptosis, which occurred presumably via suppressing activation of the components of the mitochondrial apoptotic pathway [12]. However, it is still to be examined whether shock wave therapy is also effective at attenuating cardiomyocyte apoptosis after AMI and what the potential mechanisms are. In this study, we used a rat model of AMI to examine whether CSWT could attenuate cardiomyocyte apoptosis after AMI in the border area of infarcted myocardium.

\section{Materials and Methods}

Ethics

The Animal Use and Care Committee of Beijing Hospital approved the present study. Operations and animals care were performed according to the Guide for the Care and Use of Laboratory Animals (NIH Publication \#85-23, revised 1996). 


\section{Cellular Physiology Cell Physiol Biochem 2018;49:1734-1746

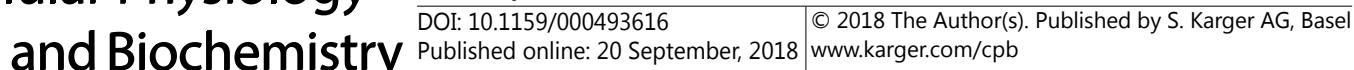

\section{Animal models}

Male Wistar rats (aged 7-8 weeks and weighing 220-250 g) were bred in climate-controll condition with 12-hour (h) light/dark cycles. The rats were received standard rat chow and water ad libitum.

Induction of MI was operated in rats by ligation of the left anterior descending coronary artery (LAD) as depicted in detail elsewhere [13-15]. In brief, animals were anesthetized with intra-peritoneal injections of pentobarbital $(30 \mathrm{mg} / \mathrm{kg}$ ) during surgery. The rats were ventilated by positive pressure with a smallanimal respirator. With a left-sided thoracotomy parallel to the sternum under aseptic conditions, the third intercostal space was carefully opened. The third and fourth ribs were separated to expose the heart. Followed by opening pericardium, the LAD was ligated with a 6-0 silk suture. The sham-operated groups underwent thoracotomy only without LAD ligation. Regional myocardial infarction was identified with an electrocardiograph.

\section{Cardiac shock wave therapy}

Based on the results of our studies, low-energy SW therapy was applied to the SW groups $12 \mathrm{~h}$ after AMI with 800 shots at $0.1 \mathrm{~mJ} / \mathrm{mm}^{2}$ to the whole heart under anesthesia. The shock wave was produced with the CSWT system (Duolith VET, Storz Medical, Switzerland). The rats in the non-SW group underwent the same procedures but without SW.

\section{Groupings}

Exploration of the appropriate time point for CSWT after AMI. Rats were randomly divided into normal control (NC) (n=5) and AMI (n=5 for each time point) groups for the following time points: $6 \mathrm{~h}, 12 \mathrm{~h}, 24 \mathrm{~h}$, $36 \mathrm{~h}$ and $48 \mathrm{~h}$ post coronary ligation.

Exploration of the optimal parameter of CSWT after AMI. Rats were randomly divided into sham group $(n=5)$, AMI group $(n=5), 600$ shots $(n=5), 800$ shots $(n=5)$ and 1000 shots $(n=5)$ of shock waves after AMI groups.

Assessment of the effects of CSWT on apoptosis of cardiomyocytes after AMI. Rats were randomly divided into four groups: sham-operated only (SHAM, n=5), sham-operated with SW (SHAM+SW, n=5), AMI only (AMI, n=6), AMI treated with SW (AMI+SW, n=6).

\section{Specimen collection}

Animals were sacrificed $36 \mathrm{~h}$ after AMI with an intravenous overdose of potassium chloride. Following the heart removal from each animal, heart chambers were repeated rinsing with normal saline to clear the red blood cells rapidly. After euthanasia, the whole heart was sliced into three cross-sections (2-mm thick slices) from the apex towards the atrioventricular groove. Consecutive slices were collected for Western blot analysis, Hematoxylin-eosin (H\&E) staining and terminal deoxynucleotidyl transferase dUTP nick-end labeling (TUNEL) assay.

\section{$H \& E$ staining}

The heart sections of the level of papillary muscle were stored in 10\% neutral formalin liquid for $24 \mathrm{~h}$. Following paraffin-embedding and transverse serial sectioning of LV slices, H\&E staining was performed for general morphological evaluations.

\section{Isolation of mitochondria for cytochrome c}

All procedures were based on the Qproteome Mitochondria Isolation Handbook (QIAGEN). In brief, tissue from the infarct border zone of the LV myocardium was added to Lysis Buffer with a 1/100 volume of a Protease Inhibitor Solution. Samples were homogenized and then incubated on an end-over-end shaker for $10 \mathrm{~min}$ at $4^{\circ} \mathrm{C}$. The homogenate was centrifuged at $1000 \mathrm{~g}$ for $10 \mathrm{~min}$ at $4^{\circ} \mathrm{C}$. The cell pellet was resuspended after removing the supernatant with ice-cold Disruption Buffer with a 1/100 volume of a Protease Inhibitor Solution. Centrifuging at $10000 \mathrm{~g}$ at $4^{\circ} \mathrm{C}, 10 \mathrm{~min}$, supernatant was centrifuged at 6000 $\mathrm{g}$ for $10 \mathrm{~min}$ at $4^{\circ} \mathrm{C}$. After removal of the supernatant, mitochondrial pellet was washed with Mitochondria Storage Buffer. Followed by centrifuging at $6000 \mathrm{~g}$ at $4^{\circ} \mathrm{C}, 20 \mathrm{~min}$, mitochondrial pellet was resuspended in the Mitochondria Storage Buffer and stored at $-80^{\circ} \mathrm{C}$. 


\section{Cellular Physiology Cell Physiol Biochem 2018;49:1734-1746 and Biochemistry \begin{tabular}{l|l} 
DOI: 10.1159/000493616 & (c) 2018 The Author(s). Published by S. Karger AG, Basel \\
www.karger.com/cpb
\end{tabular}

\section{Western blot analysis}

Protein extracts from the border areas of the infarct LV myocardia were separated by SDS-PAGE using $12 \%$ acrylamide gradients followed by transferring to PVDF membranes (Millipore). The membranes were incubating with monoclonal primary antibodies (1:1000) overnight at $4^{\circ} \mathrm{C}$ after blocking with buffer $(5 \%$ nonfat milk). The blots were incubated with HRP-conjugated secondary antibodies (1:5000) for $1 \mathrm{~h}$ at room temperature. Image s software was used to perform densitometry analysis.

\section{TUNEL Assay for Apoptotic Nuclei}

TUNEL staining and DAPI were used to detect nuclear fragmentation following the manufacturer's guidelines (Roche). A total of 2000-3000 cells in 2-3 chosen randomly fields in the border areas of the infarct myocardia from each microslide were counted to semi-quantitatively identify the ratio of the apoptotic nuclei $[16,17]$.

\section{Establishment of the CSWT methodology in the rat model of AMI}

The heart samples of the rat models were collected at $6 \mathrm{~h}, 12 \mathrm{~h}, 24 \mathrm{~h}, 36 \mathrm{~h}$ and $48 \mathrm{~h}$ after AMI, and the protein levels of Bax, Bcl-2 and cleaved-Caspase- 3 in the border zones of the infarcted myocardia were analyzed by Western blotting. The apoptosis rate of the myocardial cells was measured using the TUNEL assay.

The rat models of AMI were treated with shock waves $(600,800$, or 1000 shots $) 12 \mathrm{~h}$ after AMI. The protein levels of Bax,Bcl-2 and cleaved-Caspase-3 in the border zones of the infarcted myocardia were analyzed by Western blot.

\section{Statistical analysis}

The data are expressed as the mean \pm SD. One-way ANOVA with bonferroni correction was performed for multiple comparation. Statistical analyses were performed using the SAS statistical software for Windows version 9.2. A probability value $<0.05$ was considered statistically significant.

\section{Results}

The rat model of $A M I$

Following ligation of the left coronary artery, the immediate changes are shown, such as sudden pallor and subsequent cyanosis, marked dilatation and paralysis of the affected portion of LV (Fig. 1).

\section{Apoptosis of cardiomyocytes at} different time points after AMI

The apoptosis rates of cardiomyocytes at $6 \mathrm{~h}, 12 \mathrm{~h}, 24 \mathrm{~h}, 36 \mathrm{~h}$ and $48 \mathrm{~h}$ after ligation procedures by TUNEL assay were $4.31 \%, 8.16 \%$, $19.96 \%, 30.96 \%$, $48.59 \%$, and $69.28 \%$, respectively. The apoptosis rate at 12 $\mathrm{h}$ after AMI significantly increased compared with $\mathrm{NC}$ group $(\mathrm{P}<0.01)$ and significantly decreased compared with the $24 \mathrm{~h}$ and $36 \mathrm{~h}$ post AMI groups $(\mathrm{P}<0.01)$ (Fig. 2). Therefore, we performed SW therapy at $12 \mathrm{~h}$ after AMI. Meanwhile, we found a steady increase in the expression levels of

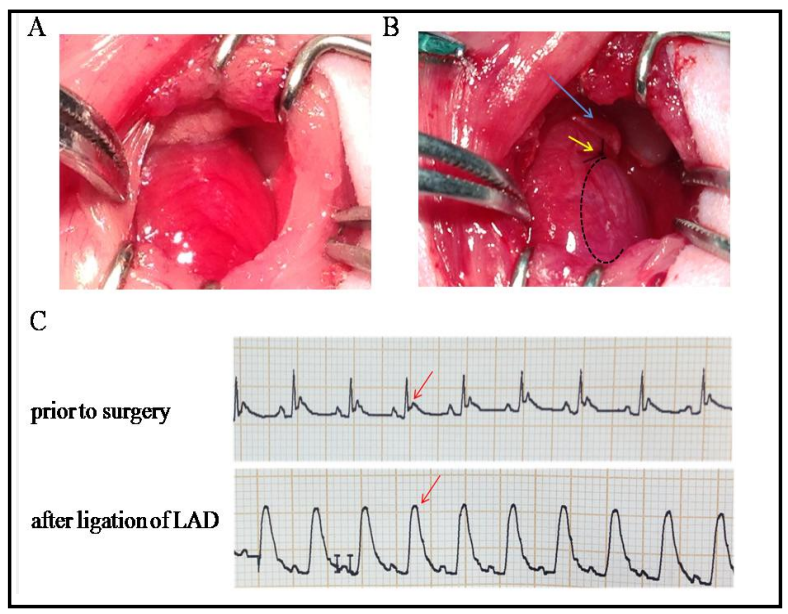

Fig. 1. Transmural MI was induced in rats by ligation of LAD. A: The heart of pre-operative. B: The immediate changes including sudden pallor and subsequent cyanosis after ligation of LAD, Blue arrow indicates left atrial appendage. Yellow arrow indicates ligature. Dotted line indicates infarcted area. C: ECG recorded in animals before and after ligation of LAD. Above - ECG recorded before ligation of LAD; Below - impaired heart rhythm in 30 minutes after ligation of LAD, red arrow indicates ST segment which is lifting above the isoelectric line after ligation of LAD. 
Bax and cleaved-Caspase-3 and a gradual decrease in the expression levels of Bcl-2 from $6 \mathrm{~h}$ to $48 \mathrm{~h}$ after coronary artery occlusion. The results showed that, as the exposure time to acute ischemia ongoing, cardiomyocyte apoptosis rate was increasing associated with the rise in proapoptosis protein expression and the reduction in anti-apoptosis protein expression in the border myocardium of MI. (Fig. 3).

The optimal parameter for CSWT after AMI

Our results showed that expression of Bcl-2 significantly increased and that expression of Bax and cleaved-Caspase-3 significantly decreased in all SW groups compared with the AMI only group. These results preliminarily showed the antiapoptosis effect of CSWT on cardiomyocytes. In the 800shot protocol, the expression level of $\mathrm{Bcl}-2$ showed the highest increase, while the expression levels of Bax and cleaved-Caspase-3 showed the strongest decrease, indicating the maximum effect of apoptosis inhibition (Fig. 4). Thus, we chose 800 shots as the treatment condition for subsequent study.

Fig. 4. Expression of apoptosisrelated protein of cardiomyocyte with different dose of shock wave $(600,800$, 1000 shots respectively) after AMI respectively. (A): Western blotting was used to analysis of Bcl-2, Bax, Caspase-3 abundance in cardiomyocyte. (B, C, D): The average data of Bcl-2, Bax, Caspase-3 in cardiomyocyte. Results are expressed as mean \pm SEM ( $\mathrm{n}=5$ each). ${ }^{* *} \mathrm{P}<0.01$ vs sham, ${ }^{\#}<0.05$, "\# $<0.01$ vs AMI.
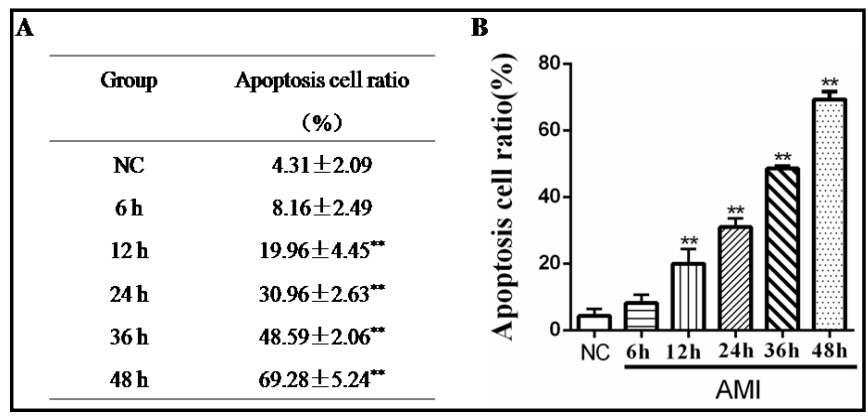

Fig. 2. Apoptosis rate of cardiomyocyte at $6 \mathrm{~h}, 12 \mathrm{~h}, 24 \mathrm{~h}, 36 \mathrm{~h}$ and $48 \mathrm{~h}$ after AMI respectively. (A, B): The average data of apoptosis ratio of cardiomyocyte (apoptosis ratio=TUNEL ${ }^{+}$nuclei nmber/DAPI ${ }^{+}$nuclei number $\left.\times 100 \%\right)$. Results are expressed as mean \pm SEM $(n=5$ each $) .{ }^{* *} \mathrm{P}<0.01$ vs NC.
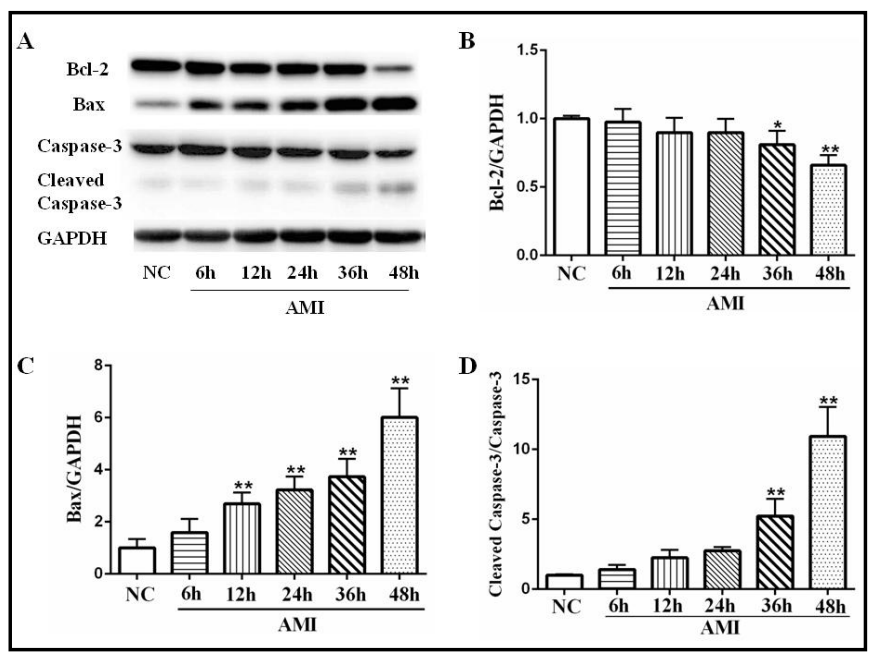

Fig. 3. Expression of apoptosis-related protein of cardiomyocyte at $6 \mathrm{~h}, 12 \mathrm{~h}, 24 \mathrm{~h}, 36 \mathrm{~h}$ and $48 \mathrm{~h}$ after AMI respectively. (A): Western blotting was used to analysis of Bcl-2, Bax, Caspase-3 abundance in cardiomyocyte. (B, C, D): The average data of Bcl2 , Bax, Caspase-3 in cardiomyocyte. Results are expressed as mean \pm SEM ( $\mathrm{n}=5$ each). ${ }^{*} \mathrm{P}<0.05{ }^{* *} \mathrm{P}<.01$ vs NC.

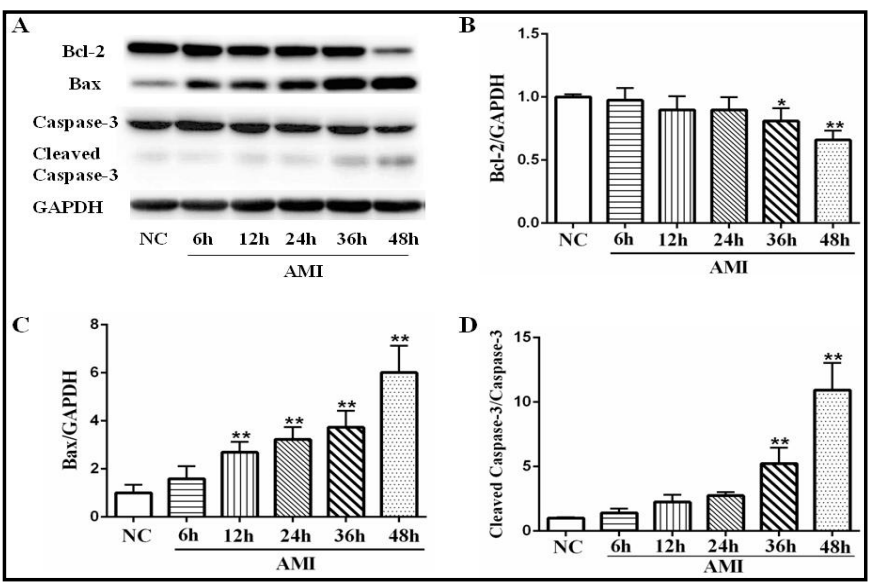




\section{Cellular Physiology Cell Physiol Biochem 2018;49:1734-1746

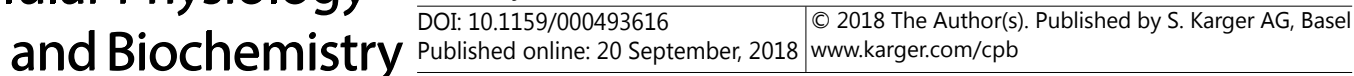

Effects of CSWT on cardiomyocyte apoptosis after $A M I$

CSWT decreased the cardiomyocyte apoptosis rate in the TUNEL assay. The apoptosis rates by TUNEL staining in the sham group, AMI group and AMI+SW group were $7.52 \pm 1.24 \%, 48.92 \pm 5.13 \%$, and $25.33 \pm 3.55 \%$, respectively. The apoptosis rate significantly increased in the AMI group compared with sham group $(\mathrm{P}<0.01)$ and was significantly reduced $(\mathrm{P}<0.01)$ after the $\mathrm{SW}$ therapy (Fig. 5). The results indicated that CSWT could reduce early apoptosis of cardiomyocytes after AMI.

CSWT inhibited mitochondrial cytochrome $c$ release into the cytoplasm. To determine whether the mitochondria-mediated apoptosis pathway induced by AMI was suppressed after SW, the spatial localization of cytochrome $c$ in cardiomyocytes was determined. The Western blot analysis revealed that mitochondrial cytochrome $\mathrm{c}$ in the AMI group significantly decreased and that cytoplasmic cytochrome c increased significantly compared with the sham and AMI+SW groups (Fig. 6). Differences were statistically significant. These findings indicated that CSWT could improve the cardiomyocyte mitochondrial membrane integrity and inhibit the movement of cytochrome $\mathrm{c}$ from the mitochondria to the cytoplasm after AMI.

CSWT suppressed the expression of apoptosis molecules. Western blotting revealed that the expression level of Bcl-2, an index of antiapoptosis, in the AMI group was substantially lower than in the sham and AMI+SW groups. Conversely, the expression levels of Bax and cleaved-Caspase-3, two indicators of apoptosis, in the AMI group were notably higher than in the sham and AMI+SW groups (Fig. 7). Differences were statistically significant. The results showed that CSWT could up-regulate the expressionof Bcl-2 and down-regulate the expression of Bax and cleaved-Caspase- 3 incardiomyocytes after AMI, which implied that CSWT was anti-apoptotic.

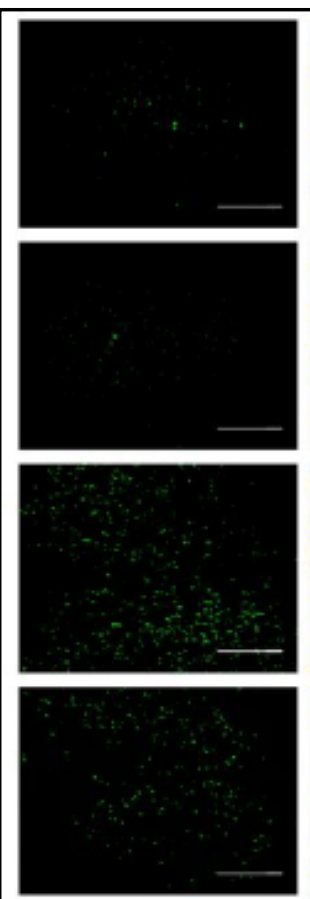

$\Delta$

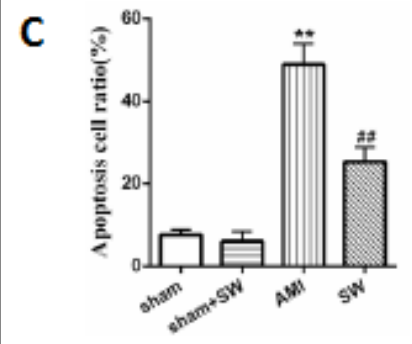

Fig. 5. CSWT attenuates apoptosis rate of cardiomyocyte. (A): TUNEL staining (200x) of apoptotic nuclei. (B): DAPI staining (200x) of total nuclei. (C): The average data of apoptosis ratio of cardiomyocyte (apoptosis ratio $=\mathrm{TUNEL}^{+}$nuclei nmber/DAPI ${ }^{+}$nuclei number $\times 100 \%)$. Scale bar represents $200 u m$. Results are expressed as mean \pm SEM (sham group: $n=5$ each, AMI group: $n=6$ each). ${ }^{* *}$ $\mathrm{P}<0.01$ vs sham, ${ }^{\# \#} \mathrm{P}<0.01$ vs AMI.

The JNK pathway maybe involved in the CSWT effects of apoptosis. The Western blot analysis revealed that compared with the sham and AMI+SW groups, phosphorylation of JNK in the AMI group significantly increased, Differences were statistically significant (Fig. 8). We also detected other apoptosis-, inflammation-, and survival-related signaling pathways, such as the p38MAPK signaling pathway. The results showed that there were no significant differences in phosphorylation of p38MAPK between the AMI and AMI+SW groups (Fig. 8). The finding indicated that CSWT might decrease cardiomyocyte apoptosis through inhibition of the JNK signaling pathway after AMI and further protect cardiomyocytes.

\section{KARGER}


Fig. 6. CSWT could inhibit the release of cytochrome $\mathrm{c}$ from mitochondrial to cytoplasm significantly. (A,C): Western blotting was used to analysis of mitochondrial cytochrome c and cytoplasmic cytochrome c abundance in cardiomyocyte. (B, D): The average data of mitochondrial cytochrome c and cytoplasmic cytochrome c in cardiomyocyte. Results are expressed as mean \pm SEM (sham group: $\mathrm{n}=5$ each, AMI group: $\mathrm{n}=6$ each). ${ }^{* *} \mathrm{P}<0.01$ vs sham, ${ }^{\# \#} \mathrm{P}<0.01$ vs AMI.

Fig. 7. CSWT could upregulate the expression of $\mathrm{Bcl}-2$ and downregulate the expression of Bax and Cleaved-Caspase-3 with statistically significant differences. (A): Western blotting was used to analysis of Bcl-2, Bax, Caspase-3 abundance in cardiomyocyte. (B, C, D): The average data of Bcl-2, Bax, Caspase-3 in cardiomyocyte. Results are expressed as mean \pm SEM (sham group: $\mathrm{n}=5$ each, AMI group: $\mathrm{n}=6$ each). ${ }^{* *} \mathrm{P}<0.01$ vs sham, ${ }^{\# \#} \mathrm{P}<0.01$ vs AMI.

Fig. $\quad 8$ Apoptosis, inflammation, and survival related signaling pathways of cardiomyocyte were activated after AMI and the anti-appototic effects of CSWT were mainly mediated through JNK-dependent signaling pathway. (A,C): Western blotting was used to analysis of JNK, p38MAPK abundance in cardiomyocyte. $(\mathrm{B}, \mathrm{D})$ : The average data of JNK, p38MAPK in cardiomyocyte. Results are expressed as mean \pm SEM (sham group: $\mathrm{n}=5$ each, AMI group: $\mathrm{n}=6$ each). ${ }^{* *} \mathrm{P}<0.01$ vs sham, ${ }^{\# \#} \mathrm{P}<0.01$ vs AMI.
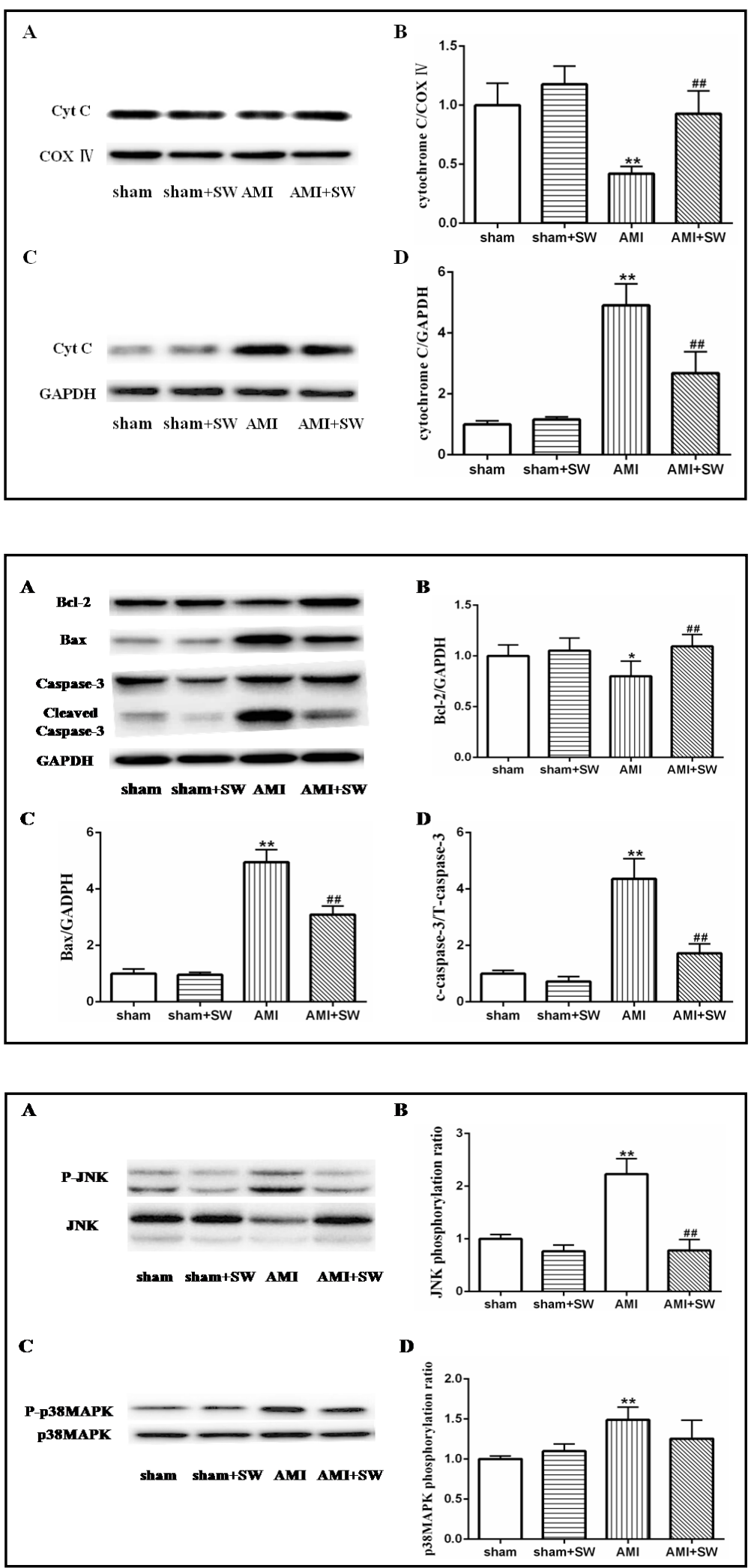


\section{Cellular Physiology Cell Physiol Biochem 2018;49:1734-1746 and Biochemistry \begin{tabular}{c|c|c|} 
DOI: 10.1159/000493616 & $\begin{array}{l}\text { O } 2018 \text { The Author(s). Published by S. Karger AG, Basel } \\
\text { Puww.karger.com/cpb }\end{array}$
\end{tabular} \\ Zhang et al.: CSWT Attenuates Cardiomyocyte Apoptosis}

\section{Histopathological study}

Hematoxylin and eosin staining revealed that the myocardial fibers were disordered with vacuolation degeneration, coagulation necrosis in the cardiomyocyte and infiltration of inflammatory cells in the AMI group compared with the sham group. Similar changes were observed in the AMI+SW group. The histopathological analysis did not show significant improvements in the above changes of shock wave therapy after AMI (Fig. 9).

\section{Discussion}

This study, which describes the impact of CSWT on the early stage of apoptosis in cardiomyocytes after AMI, has several implications. First, the apoptosis rates of cardiomyocytes at different time points (6 h-48 h) after coronary artery occlusion were estimated. The results showed that the apoptosis rates of cardiomyocytes increased with prolongation of coronary artery occlusion. Second, the dose-effect relationship for CSWT on early-stage apoptosis was observed, and the data showed that a dose of 800 shots provided the maximum effect on apoptosis inhibition. Third, and importantly, CSWT substantially attenuated myocyte apoptosis after AMI in rats through an intrinsic mitochondrial pathway. To the best of our knowledge, this is the first study to demonstrate the early-stage anti-apoptotic impacts of shock wave therapy on cardiomyocytes after AMI in vivo.

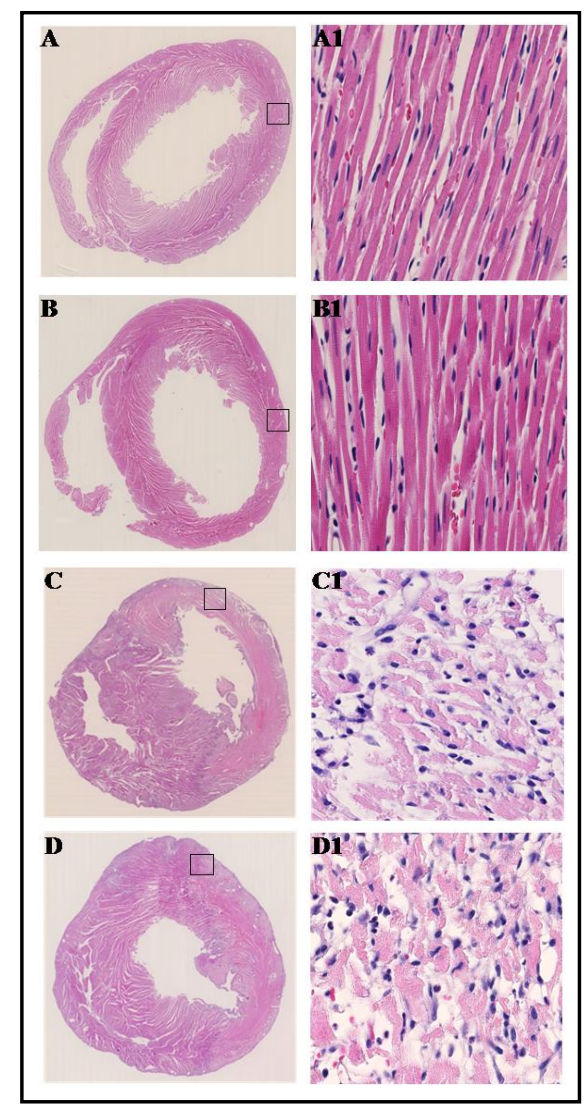

Fig. 9. Hematoxylin and Eosin staining in LV myocardium. A: sham group, B: sham+SW roup, $\mathrm{C}$ : AMI group, $\mathrm{D}$ : $\mathrm{AMI}+\mathrm{SW}$ group. A1, B1, C1, D1: Magnification of $400 \times$.

\section{Methodology of CSWT in the AMI rat model}

Apoptosis of cardiomyocytes at different time points after AMI. As a non-renewable population, maintenance of the cardiomyocyte number is crucial to preserve the structural integrity and function of the heart. Apoptosis, autophagy, and necrosis are currently defined as three modes of cell death by the morphological criteria. As a highly regulated cell deletion process, apoptosis has been put down to a pathogenic role in ischemia, hypoxia and ischemia-reperfusion in the heart. Genetic approaches have indicated that suppression of cardiomyocyte apoptosis could reduce the infarct size by $50 \%$ to $70 \%$ and ameliorate cardiac dysfunction after ischemia $[18,19]$.

Studies indicate that apoptotic cardiomyocyte become apparent at $3 \mathrm{~h}$ after LAD occlusion, peak at 1-2 days, and decrease at 7, 14, and 30 days thereafter [20]. Following the acute events, the levels of apoptosis in the border area adjacent to the infarcted myocardium rise steadily, while the levels of apoptosis apparently decrease in the infarcted area [21, 22]. With the above phenomena, we observed the apoptosis rate and expression of apoptosisrelated proteins in cardiomyocytes of the border zone adjacent to the infarcted myocardium from $6 \mathrm{~h}$ to $48 \mathrm{~h}$ after AMI. TUNEL staining shows that apoptosis of cardiomyocytes is triggered upon MI, based on Bax, cleaved-Caspase- 3 and Bcl-2 expression. We also found that apoptosis of cardiomyocytes had started but had not yet reached its peak at $12 \mathrm{~h}$ after AMI and that the pro-apoptotic signaling pathways were activated remarkably. Therefore, we started shock wave therapy on the rat model of AMI at $12 \mathrm{~h}$ after AMI, which was the appropriate time point for CSWT after AMI.

\section{KARGER}


The optimal parameter of CSWT after AMI. It has been reported that there is a dose-effect relationship in CSWT. The recommended protocol developed by Tohoku University of Japan with an energy level of $0.09 \mathrm{~mJ} / \mathrm{mm}^{2}$ and 200 shots to each point is carried out in current clinical trials [23]. However, little is known about the appropriate dose of shock wave therapy to animal model. It has been reported that an energy level ranging from $0.10-0.13 \mathrm{~mJ} / \mathrm{mm}^{2}$ and a shock number ranging from 200-300 impulses are the optimal CSWT parameters for anti-apoptosis in vitro [24]. An in vivo study indicates that low-energy SW (600 shots, $0.1 \mathrm{~mJ} / \mathrm{mm}^{2}$ ) improves post-MI LV remodeling associating with anti-inflammatory effects [25]. Based on these promising results, we chose $0.1 \mathrm{~mJ} / \mathrm{mm}^{2}$ as the shock wave energy level and examined the expression levels of apoptosis-related proteins with different wave pulses (600, 800, or 1000 shots) after AMI in vivo. We found that the expression level of Bcl2 was significantly increased and that the expression levels of Bax and cleaved-Caspase- 3 were significantly decreased in all CSWT groups, demonstrating the anti-apoptosis effect of CSWT on cardiomyocytes. The maximal effects on increasing the expression level of Bcl-2 and decreasing the expression levels of Bax and cleaved-Caspase- 3 were observed using 800 shots. Our results indicate that low-energy SW therapy $\left(0.1 \mathrm{~mJ} / \mathrm{mm}^{2}\right)$ with 800 shots exerts the preferred anti-apoptosis effect after AMI. Therefore, we chose 800 shots as the optimal parameter for shock wave therapy.

Mechanisms of the suppression effects of CSWT on cardiomyocyte apoptosis after AMI in rats. It has been previously demonstrated that CSWT improves LV function and inhibits LV remodeling [26] by enhancing angiogenesis [11, 27-29], attenuating the inflammatory response [9, 30, 31] and reducing oxidative stress [9] in the ischemic heart in vivo. However, the role of the CSWT effect on cardiomyocyte apoptosis after AMI remains to be elucidated. Our previous in vitro study revealed that CSWT attenuated ischemia and hypoxia-induced H9c2 cell apoptosis [12]. In the current study, a similar result is shown in the TUNEL staining data. We find that the apoptosis rate of the cardiomyocytes is significantly increased after AMI and remarkably reduced after SW therapy, which indicate that CSWT could reduce early apoptosis of cardiomyocytes after AMI.

Evidence exists that an intrinsic mitochondrial pathway plays a vital role in cardiomyocyte apoptosis. Cytochrome $\mathrm{c}$ is the earliest identified apoptogen of the mitochondrial pathway. It has been shown that apoptosis induced by ischemia and hypoxia is associated with translocation of cytochrome c from the mitochondria to the cytosol. One important finding in our study is a significant decrease in mitochondrial cytochrome $c$ and a remarkable increase in the level of cytoplasmic cytochrome c after AMI. Opposite trend is observed after shock wave therapy which demonstrates that CSWT can significantly inhibit translocation of cytochrome $\mathrm{c}$ from mitochondria to cytoplasm. This result implies that CSWT can inhibit the mitochondrial pathway of apoptosis by improving cardiomyocyte mitochondrial membrane integrity after AMI with decreased permeability of cytochrome c.

The Bcl-2 family of proteins mostly regulates the initiation phase of the mitochondrial pathway. Bcl-2, an index of the anti-apoptotic proteins, and Bax, an index of the pro-apoptotic proteins, play direct roles in the release of mitochondrial apoptogens such as cytochrome c [32]. Study in an ischemia-reperfusion injury mice model shows that gain-of-function mutations in Bcl-2 and loss-of-function mutations in Bax seem to decrease the infarct size by nearly $50 \%$ [33]. Analogous results have been found using pharmacological methods with protease inhibitors of multiple caspases [34-36]. Fu et al. [9] have reported that CSWT effectively increases Bcl-2 mRNA expression and decreases Bax mRNA expression that is associated with improvement of LV function in a pig model, which implies that CSWT can suppress cardiomyocyte apoptosis by regulating the Bcl-2 family of proteins. However, the precise mechanisms through which CSWT exerts anti-apoptotic actions after AMI are still unclear. Another important finding of the present study is that after AMI, the protein expression level of Bcl-2 is significantly decreased and the expression levels of Bax and cleaved-Caspase-3 are significantly increased. CSWT can strongly up-regulate the level of

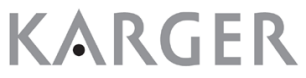




\section{Cellular Physiology Cell Physiol Biochem 2018;49:1734-1746 \\ and Biochemistry Published online; 20 September, $2018 \begin{aligned} & \text { DO 2018 The Author(s). Published by S. Karger AG, Basel } \\ & \text { www.karger.com/cpb }\end{aligned}$ \\ Zhang et al.: CSWT Attenuates Cardiomyocyte Apoptosis}

Bcl-2 and down-regulate the levels of Bax and cleaved-Caspase-3, a downstream mediator of the apoptosis pathway. Our results demonstrate that CSWT can inhibit an intrinsic mitochondrial pathway of apoptosis after AMI.

To further exposit the regulatory mechanism through which CSWT improve cardiomyocyte apoptosis after AMI, both the JNK and p38MAPK signaling pathways, which are involved in apoptosis, inflammation and cell survival, were assessed at the same time. JNK, a member of the mitogen-activated protein kinase (MAPK) family, has been suggested to have ananti-apoptosis effect on reactive oxygen species (ROS) and ischemia-induced apoptosis $[37,38]$. We found that phosphorylation of JNK and p38MAPK were significantly increased after AMI. CSWT attenuated JNK phosphorylation, but no significant differences were observed for p38MAPK phosphorylation. These results suggest that the apoptosis-, inflammation-, and cell survival-related signaling pathways of cardiomyocytes are activated after AMI and that the anti-apoptotic effects of CSWT might be mainly mediated through a JNK-dependent signaling pathway.

In addition to the molecular mechanisms, the histopathological changes have been explored. We note with regret that no significant improvements in the histopathology were evident regarding degeneration, necrosis and the inflammatory response in the ischemic heart tissue after the shock wave therapy. Abe et al. [25] found that shock wave therapy significantly ameliorated the infiltration of neutrophils on days 3 and 6 after AMI in rats. Therefore, we speculate that the anti-inflammatory effects and improvements in the histopathology after shock wave therapy lag behind the attenuation of apoptosis during the acute phase of MI.

Assessment of safety of the CSWT application. As a new and non-invasive treatment strategy for ischemic heart disease, the safety of CSWT is still controversial. Sergei [39] considers shock wave therapy to be an unselective damaging factor that would injure cell membranes and the cytoskeleton. To assess the safety of CSWT on cardiac tissue function and structure, Di et al. [40] deliver low-energy shock waves (100 shots, $\left.0.25 \mathrm{~mJ} / \mathrm{mm}^{2}\right)$ to normal Fisher 344 rats. The results show that CSWT does not elevate the cTnI level or induce arrhythmia. Histological analysis reveals neither differences in the content of extracellular matrix collagen nor the content of fibrosis in the treated area. Moreover, the shock wave does not contribute to apoptosis of the cardiomyocyte in vivo.

Our previous study on the safety of CSWT with normal Wistar rats demonstrated a lack of obvious apomorphosis, necrosis, or inflammatory cell invasion in SW-treated hearts. The TUNEL staining showed no significant increase in the cardiomyocyte apoptosis rate after CSWT. Compared with the normal control group, there was no significant differences in the expression levels of apoptosis-related proteins in the SW group [41]. To further explore the safety of shock wave therapy, the sham with SW group was designed in our study. The results showed that significant differences were not present between the cardiomyocyte apoptosis rates and between the expression levels of the apoptosis-related proteins in the sham and sham with SW groups. These results demonstrate that non-invasive CSWT is a safe technology.

\section{Conclusion}

We demonstrate that CSWT can alleviate AMI-induced cardiomyocyte apoptosis by improving cardiomyocyte mitochondrial membrane integrity, increasing the expression level of anti-apoptosis protein Bcl-2 and decreasing the expression levels of pro-apoptosis proteins Bax and cleaved-Caspase-3. We also find that the JNK pathway may relate to the effects of CSWT on anti-apoptosis. CSWT is a prospective therapeutic strategy for ischemic heart disease.

This study has several limitation. First, although we have been able to illuminate that CSWT can attenuate cardiomyocyte apoptosis in vivo, further clinical trials are required. Second, the in-depth molecular mechanisms of the anti-apoptosis effects of CSWT also need to be illuminated in future studies. Third, in vivo studies have showed low rates of 


\section{Cellular Physiology Cell Physiol Biochem 2018;49:1734-1746

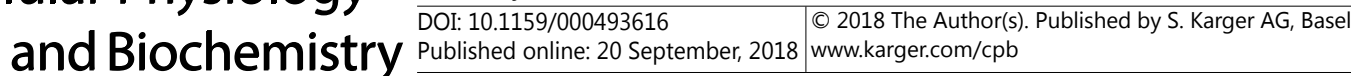 \\ Zhang et al.: CSWT Attenuates Cardiomyocyte Apoptosis}

cardiomyocyte apoptosis in heart failure. An apoptotic rate as low as $0.023 \%$, which is 5to 10 - time lessen than that of heart of patients with advanced HF, is enough to induce a deadly cardiomyopathy within 8 to 24 weeks in mice [42]. Nishida et al. [10] have found that CSWT can improve the ischemia-induced left ventricular ejection fraction in a pig model in vivo. Uwotoku et al. [11] have reported that CSWT is an effective therapy for improving left ventricular remodeling after AMI. Thus, CSWT might improve myocardial function after AMI by attenuating cardiomyocyte apoptosis. In the present study, we have not demonstrated whether the anti-apoptosis effects of shock wave therapy could improve LV function. To elucidate this issue, additional approaches, such as conditional gene knockout, will provide more theoretical foundation.

\section{Acknowledgements}

This work was supported by grants from projects supported by the Capital Health Project(Z131100004013032) and the Beijing Hospital Clinical Research 121 Project (1212016004).

\section{Disclosure Statement}

The authors declare no conflicts of interest.

\section{References}

1 Abbate A, Bussani R, Amin MS, Vetrovec GW, Baldi A: Acute myocardial infarction and heart failure: role of apoptosis. Int J Biochem Cell Biol 2006;38:1834-1840.

-2 Luz A, Santos M, Magalhaes R, Oliveira JC, Pacheco A, Silveira J, Cabral S, Torres S, Leite-Moreira AF, Carvalho H: Soluble TNF-related apoptosis induced ligand (sTRAIL) is augmented by Post-Conditioning and correlates to infarct size and left ventricle dysfunction in STEMI patients: a substudy from a randomized clinical trial. Heart Vessels 2017;32:117-125.

3 Lee Y, Gustafsson AB: Role of apoptosis in cardiovascular disease. Apoptosis 2009;14:536-548.

4 Zhang H, Wang Z, Feng SJ, Xu L, Shi HX, Chen LL, Yuan GD, Yan W, Zhuang W, Zhang YQ Zhang ZM, Dong HY: PEDF improves cardiac function in rats with acute myocardial infarction via inhibiting vascular permeability and cardiomyocyte apoptosis. Int J Mol Sci 2015;16:5618-5634.

5 Nishikido T, Oyama J, Shiraki A, Komoda H, Node K: Deletion of Apoptosis Inhibitor of Macrophage (AIM)/ CD5L Attenuates the Inflammatory Response and Infarct Size in Acute Myocardial Infarction. J Am Heart Assoc 2016;5:e002863.

6 Wu J: Shear stress in cells generated by ultrasound. Prog Biophys Mol Biol 2007;93:363-373.

7 Chen H, Brayman AA, Kreider W, Bailey MR, Matula TJ: Observations of translation and jetting of ultrasound-activated microbubbles in mesenteric microvessels. Ultrasound Med Biol 2011;37:2139-2148.

-8 Gotte G, Amelio E, Russo S, Marlinghaus E, Musci G, Suzuki H: Short-time non-enzymatic nitric oxide synthesis from L-arginine and hydrogen peroxide induced by shock waves treatment. FEBS Lett 2002;520:153-155.

-9 Fu M, Sun CK, Lin YC, Wang CJ, Wu CJ, Ko SF, Chua S, Sheu JJ, Chiang CH, Shao PL, Leu S, Yip HK: Extracorporeal shock wave therapy reverses ischemia-related left ventricular dysfunction and remodeling: molecular-cellular and functional assessment. PLoS One 2011;6:e24342.

10 Nishida T, Shimokawa H, Oi K, Tatewaki H, Uwatoku T, Abe K, Matsumoto Y, Kajihara N, Eto M, Matsuda T, Yasui H, Takeshita A, Sunagawa K: Extracorporeal cardiac shock wave therapy markedly ameliorates ischemia-induced myocardial dysfunction in pigs in vivo. Circulation 2004;110:3055-3061.

11 Uwatoku T, Ito K, Abe K, Oi K, Hizume T, Sunagawa K, Shimokawa H: Extracorporeal cardiac shock wave therapy improves left ventricular remodeling after acute myocardial infarction in pigs. Coron Artery Dis 2007;18:397-404. 


\section{Cellular Physiology Cell Physiol Biochem 2018;49:1734-1746 and Biochemistry DOI: 10.1159/000493616 2018 (O 2018 The Author(s). Published by S. Karger AG, Basel

12 Yu W, Shen T, Liu B, Wang S, Li J, Dai D, Cai J, He Q: Cardiac shock wave therapy attenuates H9c2 myoblast apoptosis by activating the AKT signal pathway. Cell Physiol Biochem 2014;33:1293-1303.

13 Ye J, Yang L, Sethi R, Copps J, Ramjiawan B, Summers R, Deslauriers R: A new technique of coronary artery ligation: experimental myocardial infarction in rats in vivo with reduced mortality. Mol Cell Biochem 1997;176:227-233.

14 Molthen RC: A simple, inexpensive, and effective light- carrying laryngoscopic blade for orotracheal intubation of rats. J Am Assoc Lab Anim Sci 2006;45:88-93.

15 Sheu JJ, Lee FY, Yuen CM, Chen YL, Huang TH, Chua S, Chen YL, Chen CH, Chai HT, Sung PH, Chang HW, Sun CK, Yip HK: Combined therapy with shock wave and autologous bone marrow-derived mesenchymal stem cells alleviates left ventricular dysfunction and remodeling through inhibiting inflammatory stimuli, oxidative stress \& enhancing angiogenesis in a swine myocardial infarction model. Int J Cardiol 2015;193:69-83.

-16 Cao Y, Ruan Y, Shen T, Huang X, Li M, Yu W, Zhu Y, Man Y, Wang S, Li J: Astragalus polysaccharide suppresses doxorubicin-induced cardiotoxicity by regulating the PI3k/Akt and p38MAPK pathways. Oxid Med Cell Longev 2014;2014:674219.

17 Cao Y, Shen T, Huang X, Lin Y, Chen B, Pang J, Li G, Wang Q, Zohrabian S, Duan C, Ruan Y, Man Y, Wang S, Li J: Astragalus polysaccharide restores autophagic flux and improves cardiomyocyte function in doxorubicininduced cardiotoxicity. Oncotarget 2017;8:4837-4848.

18 Lee P, Sata M, Lefer DJ, Factor SM, Walsh K, Kitsis RN: Fas pathway is a critical mediator of cardiac myocyte death and MI during ischemia-reperfusion in vivo. Am J Physiol Heart Circ Physiol 2003;284:H456-463.

19 Chen Z, Chua CC, Ho YS, Hamdy RC, Chua BH: Overexpression of Bcl-2 attenuates apoptosis and protects against myocardial I/R injury in transgenic mice. Am J Physiol Heart Circ Physiol 2001;280:H2313-2320.

20 Cheng W, Kajstura J, Nitahara JA, Li B, Reiss K, Liu Y, Clark WA, Krajewski S, Reed JC, Olivetti G, Anversa P: Programmed myocyte cell death affects the viable myocardium after infarction in rats. Exp Cell Res 1996;226:316-327.

-21 Kajstura J, Cheng W, Reiss K, Clark WA, Sonnenblick EH, Krajewski S, Reed JC, Olivetti G, Anversa P: Apoptotic and necrotic myocyte cell deaths are independent contributing variables of infarct size in rats. Lab Invest 1996;74:86-107.

22 Mani K, Kitsis RN: Myocyte apoptosis: programming ventricular remodeling. J Am Coll Cardiol 2003;41:761-764.

-23 Kikuchi Y, Ito K, Ito Y, Shiroto T, Tsuburaya R, Aizawa K, Hao K, Fukumoto Y, Takahashi J, Takeda M, Nakayama M, Yasuda S, Kuriyama S, Tsuji I, Shimokawa H: Double-blind and placebo-controlled study of the effectiveness and safety of extracorporeal cardiac shock wave therapy for severe angina pectoris. Circ J 2010;74:589-591.

24 Zhang X, Yan X, Wang C, Tang T, Chai Y: The dose-effect relationship in extracorporeal shock wave therapy: the optimal parameter for extracorporeal shock wave therapy. J Surg Res 2014;186:484-492.

25 Abe Y, Ito K, Hao K, Shindo T, Ogata T, Kagaya Y, Kurosawa R, Nishimiya K, Satoh K, Miyata S, Kawakami K, Shimokawa H: Extracorporeal low-energy shock-wave therapy exerts anti-inflammatory effects in a rat model of acute myocardial infarction. Circ J 2014;78:2915-2925.

-26 Kagaya Y, Ito K, Takahashi J, Matsumoto Y, Shiroto T, Tsuburaya R, Kikuchi Y, Hao K, Nishimiya K, Shindo T, Ogata T, Kurosawa R, Eguchi K, Monma Y, Ichijo S, Hatanaka K, Miyata S, Shimokawa H: Low-energy cardiac shockwave therapy to suppress left ventricular remodeling in patients with acute myocardial infarction: a first-in-human study. Coron Artery Dis 2018;29:294-300.

-27 Zimpfer D, Aharinejad S, Holfeld J, Thomas A, Dumfarth J, Rosenhek R, Czerny M, Schaden W, Gmeiner M, Wolner E, Grimm M: Direct epicardial shock wave therapy improves ventricular function and induces angiogenesis in ischemic heart failure. J Thorac Cardiovasc Surg 2009;137:963-970.

28 Holfeld J, Zimpfer D, Albrecht-Schgoer K, Stojadinovic A, Paulus P, Dumfarth J, Thomas A, Lobenwein D, Tepekoylu C, Rosenhek R, Schaden W, Kirchmair R, Aharinejad S, Grimm M: Epicardial shock-wave therapy improves ventricular function in a porcine model of ischaemic heart disease. J Tissue Eng Regen Med 2014; DOI:10.1002/term.1890.

29 Suhr F, Delhasse Y, Bungartz G, Schmidt A, Pfannkuche K, Bloch W: Cell biological effects of mechanical stimulations generated by focused extracorporeal shock wave applications on cultured human bone marrow stromal cells. Stem Cell Res 2013;11:951-964. 


\section{Cellular Physiology Cell Physiol Biochem 2018;49:1734-1746

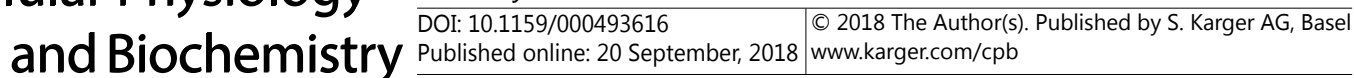 \\ Zhang et al.: CSWT Attenuates Cardiomyocyte Apoptosis}

30 Holfeld J, Tepekoylu C, Kozaryn R, Urbschat A, Zacharowski K, Grimm M, Paulus P: Shockwave therapy differentially stimulates endothelial cells: implications on the control of inflammation via toll-Like receptor 3. Inflammation 2014;37:65-70.

-31 Ciampa AR, de Prati AC, Amelio E, Cavalieri E, Persichini T, Colasanti M, Musci G, Marlinghaus E, Suzuki H, Mariotto S: Nitric oxide mediates anti-inflammatory action of extracorporeal shock waves. FEBS Lett 2005;579:6839-6845.

-32 Mani K: Programmed cell death in cardiac myocytes: strategies to maximize post-ischemic salvage. Heart Fail Rev 2008;13:193-209.

-33 Hochhauser E, Kivity S, Offen D, Maulik N, Otani H, Barhum Y, Pannet H, Shneyvays V, Shainberg A, Goldshtaub V, Tobar A, Vidne BA: Bax ablation protects against myocardial ischemia-reperfusion injury in transgenic mice. Am J Physiol Heart Circ Physiol 2003;284:H2351-2359.

34 Yoshida H, Kong YY, Yoshida R, Elia AJ, Hakem A, Hakem R, Penninger JM, Mak TW: Apaf1 is required for mitochondrial pathways of apoptosis and brain development. Cell 1998;94:739-750.

-35 Hakem R, Hakem A, Duncan GS, Henderson JT, Woo M, Soengas MS, Elia A, de la Pompa JL, Kagi D, Khoo W, Potter J, Yoshida R, Kaufman SA, Lowe SW, Penninger JM, Mak TW: Differential requirement for caspase 9 in apoptotic pathways in vivo. Cell 1998;94:339-352.

>36 Nishikawa S, Tatsumi T, Shiraishi J, Matsunaga S, Takeda M, Mano A, Kobara M, Keira N, Okigaki M, Takahashi T, Matsubara H: Nicorandil regulates Bcl-2 family proteins and protects cardiac myocytes against hypoxia-induced apoptosis. J Mol Cell Cardiol 2006;40:510-519.

37 Shen HM, Liu ZG: JNK signaling pathway is a key modulator in cell death mediated by reactive oxygen and nitrogen species. Free Radic Biol Med 2006;40:928-939.

38 Sun L, Fan H, Yang L, Shi L, Liu Y: Tyrosol prevents ischemia/reperfusion-induced cardiac injury in H9c2 cells: involvement of ROS, Hsp70, JNK and ERK, and apoptosis. Molecules 2015;20:3758-3775.

39 Jargin SV: Shock wave therapy of ischemic heart disease in the light of general pathology. Int J Cardiol 2010;144:116-117.

40 Di Meglio F, Nurzynska D, Castaldo C, Miraglia R, Romano V, De Angelis A, Piegari E, Russo S, Montagnani S: Cardiac shock wave therapy: assessment of safety and new insights into mechanisms of tissue regeneration. J Cell Mol Med 2012;16:936-942.

41 Zhang YH, Shen T, Liu B, Du L, Zhao C, He Q: Safety studies on cardiac shock wave therapy on heart. Chin J Cardiovasc Med 2016;21:126-130.

42 Wencker D, Chandra M, Nguyen K, Miao W, Garantziotis S, Factor SM, Shirani J, Armstrong RC, Kitsis RN: A mechanistic role for cardiac myocyte apoptosis in heart failure. J Clin Invest 2003;111:1497-1504. 